Poster (E29)

Gynecologic Cancer Screening

https://doi.org/10.3802/jgo.2021.32.S1.E29

\section{Profiling of menopausal symptoms and therapeutic effects of hormone replacement therapy (HRT) in endometrial cancer survivors}

\author{
Megumi Yokota," Wataru Yamagami, Shiyoko Kitazawa, \\ Satoko Tanimoto, Takuma Yoshimura, Kensuke Sakai, \\ Takayuki Chiyoda, Takashi Iwata, Koji Banno, Daisuke Aoki \\ Keio University School of Medicine, Tokyo, Japan \\ (megu_ayu2726@yahoo.co.jp)
}

Objective: This study aimed to investigate the profile of menopausal symptoms in endometrial cancer survivors and analyze the effects and risks of hormone replacement therapy (HRT). Methods: This was a retrospective study in 33 endometrial cancer survivors who underwent hysterectomy and bilateral oophorectomy (BSO) at Keio University Hospital from January 2013 to June 2019 and received HRT. We administered HRT to patients with postsurgical stage I or II endometrial cancer who complained of menopausal symptoms. Some patients with severe menopausal symptoms diagnosed with stage III disease were also treated after obtaining informed consent after being informed of the risks. Patients filled out a questionnaire on menopausal symptoms before and after HRT. We analyzed patient characteristics, clinicopathological factors, adverse events, and oncologic outcomes, which were extracted from medical records. Results: The median age at surgical menopause was 44 years, and the median body mass index was $21.2 \mathrm{~kg} / \mathrm{m}^{2}$. Using the The International Federation of Gynaecology and Obstetrics 2008 staging criteria, 30 patients were diagnosed with postsurgical stage I disease, and 3 patients were with diagnosed with stage III disease. The histological grade of endometrial cancer was grade 1 in 22 patients, grade 2 in 8 patients, and grade 3 in 2 patients. The median duration of HRT was 963 days (641-2,681 days). Our results revealed that $31 / 33$ patients $(96 \%)$ showed improvement of menopausal symptoms. During the entire observation period, there were no cases of endometrial cancer recurrence.

Conclusion: This study shows that HRT can be given for menopausal symptoms in endometrial cancer survivors in Japan.

Poster (E30)

Endometrial Hyperplasia, Endometrial Intra-epithelial Neoplasia, and Endometrial Cancer

https://doi.org/10.3802/jgo.2021.32.S1.E30

\section{Sentinel lymph node mapping in endometrial cancer}

Atthapon Jaishuen," Pisutt Srichaikul, Khemanat Khemworapong Siriraj Hospital, Bangkok, Thailand (ajaishuen@hotmail.com)

A 57-year-old female presented with postmenopausal bleeding. Fractional curettage showed grade 1 endometrioid carcinoma. She underwent laparoscopic surgical staging with sentinel lymph node (SLN) biopsy using indocyanine green (ICG) and near infrared camera. ICG was injected at 2, 4, 8, and 10 o'clock of cervix, $1 \mathrm{~mL}$ superficially and $1 \mathrm{~mL}$ at $1 \mathrm{~cm}$ depth. Ten minutes after ICG injection, the laparoscopic surgery was begun. Theoretically, ICG will come out of the cervix through the lymph chain, cross the internal iliac artery and $90 \%$ drain at obturator or external iliac LN. Otherwise, the SLN will be found in internal iliac, presacral or para-aortic area. This patient has ICG SLN at both obturator area. The patient underwent total laparoscopic hysterectomy, bilateral pelvic lymphadenectomy and para-aortic LN dissection to the level of inferior mesenteric artery. The SLNs were processed with ultrastaging, which was positive micrometastasis on the right side. The full lymphadenectomy revealed negative of other 26 pelvic LNs on the right, 18 pelvic LNs of the left and 5 para-aortic LNs. The postoperative period was uneventful. The patient was discharge in 2 days. We conducted the prospective study to see the detection rate of SLN in endometrial cancer $(20 \%$ conventional laparoscopy and $80 \%$ laparotomy). The detection rate of unilateral SLN was $99 \%$. The detection rate of bilateral SLN was $90 \%$. We performed $100 \%$ pelvic lymphadenectomy (mean LN of 17) and 80\% para-aortic LN removal (mean LN of 5). The sensitivity of SLN compared to full lymphadenectomy was $86 \%$ with $98 \%$ negative predictive value and $98 \%$ accuracy. The false negative rate was still high at $14 \%$. However, this study is not designed to achieve the false negative rate. We are recruiting more patients to get the real false negative rate. If it is acceptable, we will not do the full lymphadenectomy anymore.

\section{Ovarian cancer}

Poster (001)

Epithelial Ovarian Cancer including Borderline Tumor https://doi.org/10.3802/jgo.2021.32.S1.001

\section{A retrospective review of mucinous borderline ovarian tumor at Siriraj Hospital, Thailand: oncologic outcome including important risk factors}

Vitcha Poonyakanok, " Pattama Chaopotong, Malee Warnnissorn Siriraj Hospital, Mahidol University, Bangkok, Thailand (vpoonyakanok@gmail.com) 